\title{
Comparison of the effectiveness of extensor muscle strengthening exercise by itself, exercise with polydeoxyribonucleotide injection, and exercise with extracorporeal shockwave therapy in lateral epicondylitis: a randomized controlled trial
}

\author{
Bum Jin Shim ${ }^{1}$, Eun-Min Seo ${ }^{1}$, Jung-Taek Hwang ${ }^{1}$, Do-Young Kim ${ }^{1}$, Jae-Shin Yang ${ }^{1}$, Su-Jung Seo ${ }^{1}$, \\ Myung Sun Hong ${ }^{2}$ \\ ${ }^{1}$ Department of Orthopedic Surgery, Chuncheon Sacred Heart Hospital, Hallym University Medical College, Chuncheon, Korea \\ ${ }^{2}$ Department of Radiology, Chuncheon Sacred Heart Hospital, Hallym University Medical College, Chuncheon, Korea
}

\begin{abstract}
Background: Extensor muscle strengthening exercises with counterforce braces (EX) is a conventional conservative treatment for lateral epicondylitis (LE) of the elbow. In addition, polydeoxyribonucleotide (PDRN) or extracorporeal shockwave therapy (ESWT) has been recently used for LE.

Methods: Sixty-three patients with chronic LE participated in this study and randomly allocated in three groups (G1: EX, G2: EX+PDRN injection, and G3: EX+ESWT). All of the three groups were taught to perform EX at the first out-patient department (OPD) visit. Group 2 was injected with $3 \mathrm{~mL}$ PDRN (5.625 mg/3 mL), while group 3 received ESWT at the first OPD visit. Visual analog scale pain score, Mayo elbow performance score (MEPS), and ultrasonographic examination were checked before, 6 weeks, and 12 weeks after the treatments. Results: Overall functional scores and ultrasonographic findings in all three groups improved after treatment. The mean MEPS in group 2 improved more than groups 1 and 3 at 6 weeks (G1, 56.9>62.4; G2, 54.3>65.0; G3, 55.7>62.6), and more than group 1 at 12 weeks (G1, 56.9>67.9; G2, 54.3>73.6). The mean common extensor tendon depth (CETD) on ultrasonography in group 2 increased more than groups 1 and 3 at 6 and 12 weeks ( 6 weeks: G1, 0.385>0.386; G2, 0.332>0.392; G3, 0.334>0.357; 12 weeks: G1, 0.385>0.409; G2, 0.332>0.438; G3, $0.334>0.405[\mathrm{~cm}])$.

Conclusions: PDRN injections combined with EX exhibited a greater improvement in mean MEPS and mean CETD compared to EX only or EX combined with ESWT for LE within the 12 weeks follow-up.
\end{abstract}

Keywords: Lateral epicondylitis; Extensor muscle strengthening exercise; Counterforce brace; Polydeoxyribonucleotide; Extracorporeal shockwave therapy

Received: May 10, $2021 \quad$ Revised: July 23, $2021 \quad$ Accepted: July 26, 2021

Correspondence to: Jung-Taek Hwang

Department of Orthopedic Surgery, Chuncheon Sacred Heart Hospital, Hallym University Medical College, 77 Sakju-ro, Chuncheon 24253, Korea

Tel: +82-33-240-5197, Fax: +82-33-252-0177, E-mail: drakehjt@hanmail.net, ORCID: https://orcid.org/0000-0003-4189-084X

Co-correspondence to: Myung Sun Hong

Department of Radiology, Chuncheon Sacred Heart Hospital, Hallym University Medical College, 77 Sakju-ro, Chuncheon 24253, Korea

Tel: +82-33-240-5158, Fax: +82-33-242-7085, E-mail: mshong429@hallym.or.kr, ORCID: https://orcid.org/0000-0002-9264-2750

Bum Jin Shim and Eun-Min Seo contributed equally as co-first authors.

Financial support: None.

Conflict of interest: None.

Copyright@ 2021 Korean Shoulder and Elbow Society.

This is an Open Access article distributed under the terms of the Creative Commons Attribution Non-Commercial License (http://creativecommons.org/licenses/by-nc/4.0/) which permits unrestricted non-commercial use, distribution, and reproduction in any medium, provided the original work is properly cited. 


\section{INTRODUCTION}

Lateral epicondylitis (LE), also known as tennis elbow, is the most common cause of elbow pain in the adult population [1]. The incidence rate of LE is estimated to be four to seven per 1,000 patients per year. The majority of cases are believed to be caused by musculotendinous lesions of the common extensor tendon originating at or near the attachment to the lateral epicondyle, often as a result of overload injury and typically after minor and often unrecognized microtrauma [2]. The diagnosis is generally clinical, but in patients with persistent findings despite treatment or in patients who plan to undergo surgery, imaging may be necessary [1]. Therefore, ultrasonographic evaluation for injuries of the common extensor tendon, the cortex of the lateral epicondyle, or nearby soft tissues could be helpful $[1,2]$.

The treatment of LE varies from "wait and see," nonsteroidal anti-inflammatory drugs, physical therapies, extensor muscle strengthening exercises with counterforce braces (EX), injection therapy including glucocorticoid, polydeoxyribonucleotide (PDRN) or platelet rich plasma (PRP), and extracorporeal shockwave therapy (ESWT), and uncommonly as a last option, surgery [1-4]. Recently, PDRN injections have also been used for LE. PDRN is a tissue regeneration activator that is composed of a mixture of nucleotides and activates adenosine A2A receptors, stimulating vascular endothelial growth factor (VEGF) and the activity of fibroblasts [4-7]. ESWT, in orthopedic cases, is used to induce neovascularization at the junction of the tendon-bone, and to release growth factors. Subsequently, tissue repair is achieved through the improvement of the blood supply and increase in cell proliferation, leading to tissue regeneration of the tendon and bones [8].

Although few in number, comparisons between treatment alternatives have been studied in the literature with conflicting results and no single intervention has been proven to be the most efficient. Therefore, the object of the present study is to compare the clinical and ultrasonographic results of three treatment options: EX only, EX with PDRN injections, and EX with ESWT.

\section{METHODS}

We conducted this study in compliance with the principles of the Declaration of Helsinki. The study's protocol was reviewed and approved by the Institutional Review Board of the Chuncheon Sacred Heart Hospital (IRB no. 2013-35). All patients provided written informed consent to participate in this study.

This study was a randomized controlled trial of 69 patients who visited our out-patient department (OPD) and received a diagnosis of LE from May 2013 to April 2014. Sixty-nine patients with chronic LE were enrolled in this study and allocated in three groups (G1: EX only, G2: EX+PDRN injection, and G3: EX+ ESWT) under single-blind randomization. The randomization was performed by an independent nurse using a computerized random-sequence generator. Inclusion criteria were LE symptoms that persisted or increased for more than 3 months in which LE was defined as pain on the lateral side of the elbow and pain at the lateral epicondyle upon direct palpation and during resisted dorsiflexion of the wrist.

Exclusion criteria were ages younger than 20 years, history of ipsilateral elbow surgery, common extensor tendon tears more than $30 \%$ in depth, inflammatory diseases (e.g., rheumatoid arthritis, psoriatic arthritis), osteoarthritis with a limitation of range of motion (flexion contracture $>30^{\circ}$ and further flexion $<100^{\circ}$ ), neurological deficits in the ipsilateral upper limb, and follow-ups less than 12 weeks. The practitioners who participated in the procedure were blinded to the patient's information. According to the above criteria, six patients were excluded and 21 patients remained in each of the three group (Fig. 1). All of the three groups were taught to perform EX at the first visit in the OPD. Group 2 was injected with $3 \mathrm{~mL}$ PDRN $(5.625 \mathrm{mg} / 3 \mathrm{~mL}$ ), while group 3 received ESWT (pressure 1.5 bar, frequency 4.0 $\mathrm{Hz}$, number 500; DolorClast Master; EMS, Nyon, Swiss) on the first visit in the OPD [1]. ESWT was performed by a 7 years-experienced orthopedic nurse after the initial ultrasonographic examination and marking of the lesion. From the first OPD visit, a nonsteroidal anti-inflammatory drug (100 mg of aceclofenac) was given orally with a drug protective of the gastric mucosa (60 mg of eupatilin) twice daily. Visual analog scale (VAS) pain score, Mayo elbow performance score (MEPS), and ultrasonographic examinations were checked before, 6 weeks, and 12 weeks after the treatments. VAS pain score was based on a scale from 0 to 10 , where 0 indicated no pain and 10 represented severe pain.

The ultrasonographic evaluations were performed by a musculoskeletal radiologist with 19 years of experience who was blinded to the patient's information on the iU22 machine (Philips Healthcare, Bothell, WA, USA) using a 5- to $12-\mathrm{MHz}$ linear probe at all clinical visits. The patients were examined in a sitting position with the elbow flexed to $90^{\circ}$, the forearm neutral, and the arm resting on a pillow on the patient's thighs. A linear probe was prepared with $70 \%$ alcohol and a thin layer of sterile ultrasonographic transmission gel (Sung Heung, Bucheon, Korea). The transducer was aligned with the long axis of the radius over the common extensor tendon origin. The common extensor tendon origin was examined with color Doppler ultrasonography in the longitudinal plane by moving the transducer from side to side, 


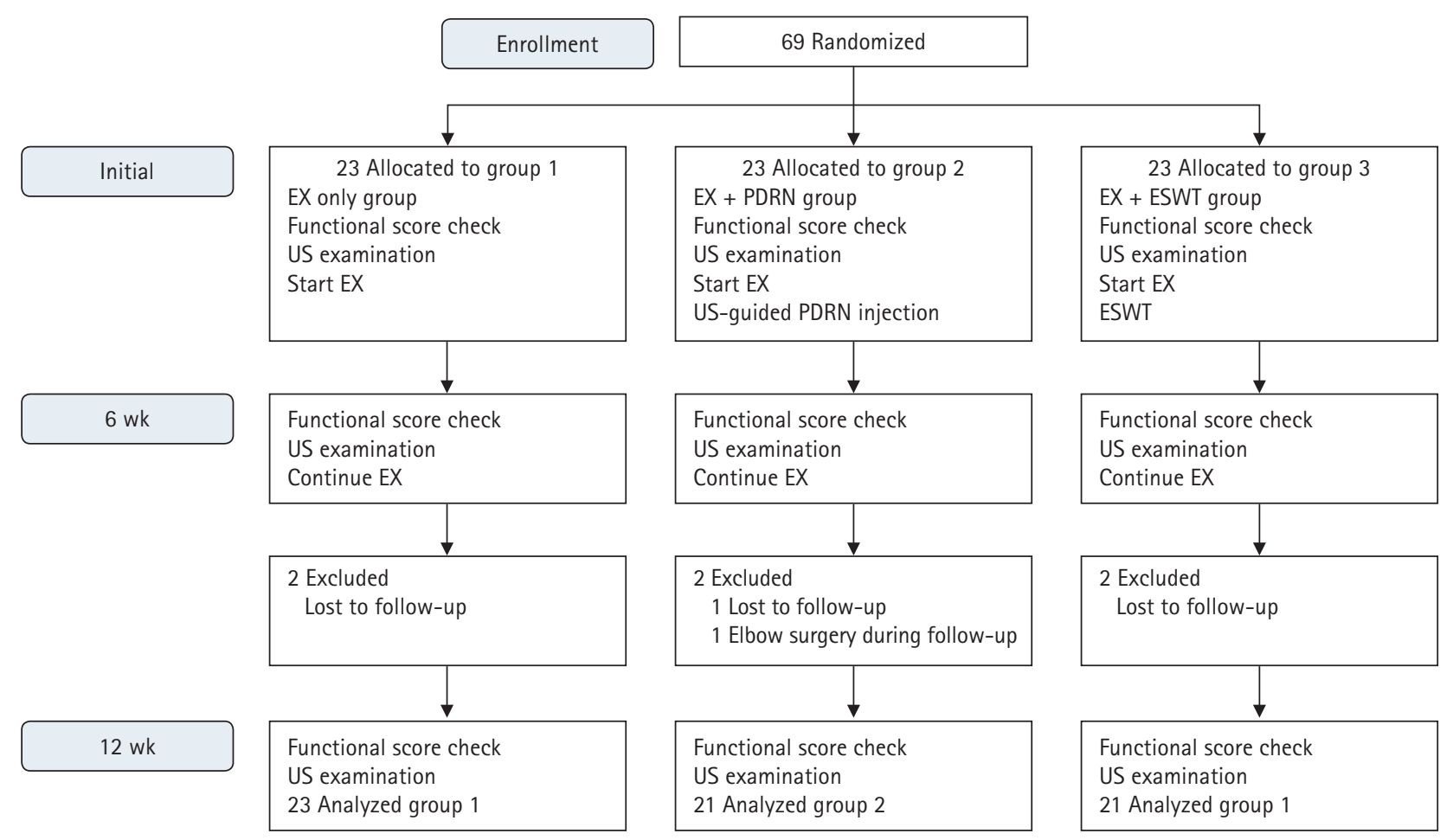

Fig. 1. Flow diagram of this study. EX: extensor muscle strengthening exercises with counterforce braces, US: ultrasound, PDRN: polydeoxyribonucleotide, ESWT: extracorporeal shockwave therapy.

locating the part with the most Doppler activity. The color Doppler activity (CDA) was observed in an area limited proximally by the tip of the lateral epicondyle and distally by the humeroradial joint space [2]. The superficial border involved the most superficial fibers, while the deep border was the bone. The CDA in the present study was ranked in a scale from 0 to 4 . The grading was estimated in a $0.5-\mathrm{cm}$ longitudinal part of the tendon with maximal Doppler activity (grade 0, no activity; grade 1, single vessel in the region of interest [ROI]; grade $2,<25 \%$; grade 3 , $25 \%-50 \%$; grade $4,>50 \%$ of the ROI [2]) (Fig. 2). Color velocity scale was set at $11.3 \mathrm{~cm} / \mathrm{sec}$. The common extensor tendon depth (CETD) was measured at an anatomic landmark at the bony surface of the lateral epicondyle, labelled the "plateau" [2]. This plateau is located at the capitellum between the insertion of the tendon and the humeroradial joint [2]. Common extensor tendon tear thickness (CETTT) was measured in a similar manner to the CETD (Fig. 3).

The ultrasound-guided PDRN injections were performed by an orthopedic surgeon blinded from the patient's information using 5- to 13-MHz linear ultrasonography transducer (Logiq p6 pro; GE Healthcare, Chicago, IL, USA) in the same position using a 5-mL syringe with $21-\mathrm{G}$ needle filled with the drug. The injection was performed with one skin portal, and the content was injected at the middle aspect, partial thickness tear site of the common extensor tendon origin, or tendon sheath if there was too much resistance [4].

The strengthening exercise of the extensor muscle with a counterforce brace was taught in all three groups. The counterforce brace was applied on the patient's forearm $2 \mathrm{~cm}$ below the lateral epicondyle, and the patient's arm was positioned on the table with the elbow flexed to $30^{\circ}$. The exercise was initiated with all the ipsilateral fingers and the ipsilateral wrist fully extended, the position maintained, and isometric force applied for 10 seconds, followed by a 10 -second rest with all the fingers slightly flexed and the wrist neutral. This was performed ten times for one set, and three sets were completed just after eating (Fig. 4) [9].

A power analysis indicated that a sample size of 54 patients (18 per group) would provide a statistical power of $80 \%$ with a two-sided alpha level of 0.05 to detect a significant difference in the improvement of VAS pain score between the initial visit and 12 weeks after treatment, assuming an effect size of 0.85 (mean difference, 1.10; standard deviation [SD], 1.29]). This was based on the mean and SD of improvement of the VAS pain score between the initial visit and 12 weeks after treatment observed in a pilot study of 20 patients. Wilcoxon signed-rank test or paired t-test according to the normality of data were completed to evaluate the differences between the initial visit and 12 weeks after treatment. One-way analysis of variance, followed by Bonferroni 

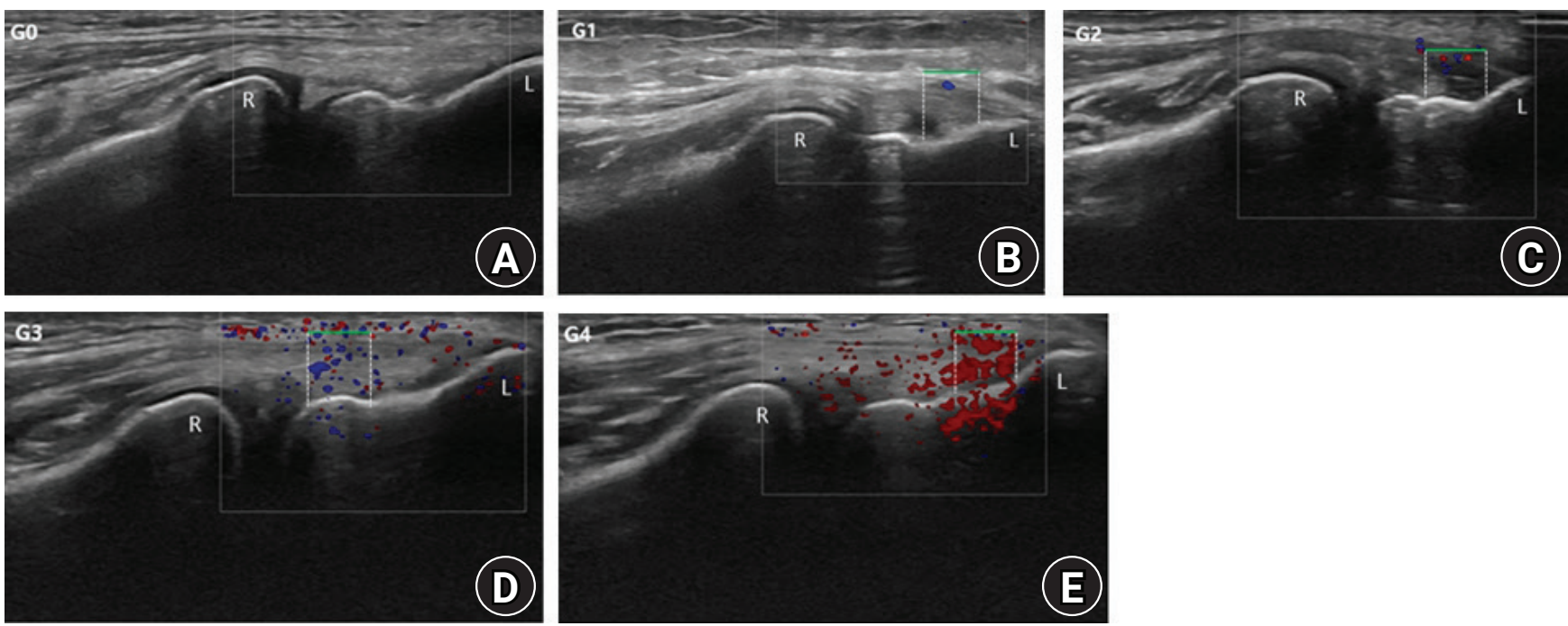

Fig. 2. The grading of the color Doppler activity (CDA) on longitudinal ultrasonographic examinations of the common extensor tendon origin. The grading was performed in the region of interest (ROI) defined as a $0.5 \mathrm{~cm}$ longitudinal part of the tendon with maximum CDA. A horizontal green line measuring $0.5 \mathrm{~cm}$ marks the superficial border of the ROI, white dotted lines mark the proximal and distal borders, and the bone marks the deep border. Flow towards the transducer is depicted in red while flow away from the transducer is shown in blue. (A) G0, no activity. (B) G1, single vessel in the ROI. (C) G2, CDA in <25\% of the ROI. (D) G3, CDA in $25 \%-50 \%$ of the ROI. (E) G4, CDA in >50\% of the ROI. R: radial head, L: lateral epicondyle.
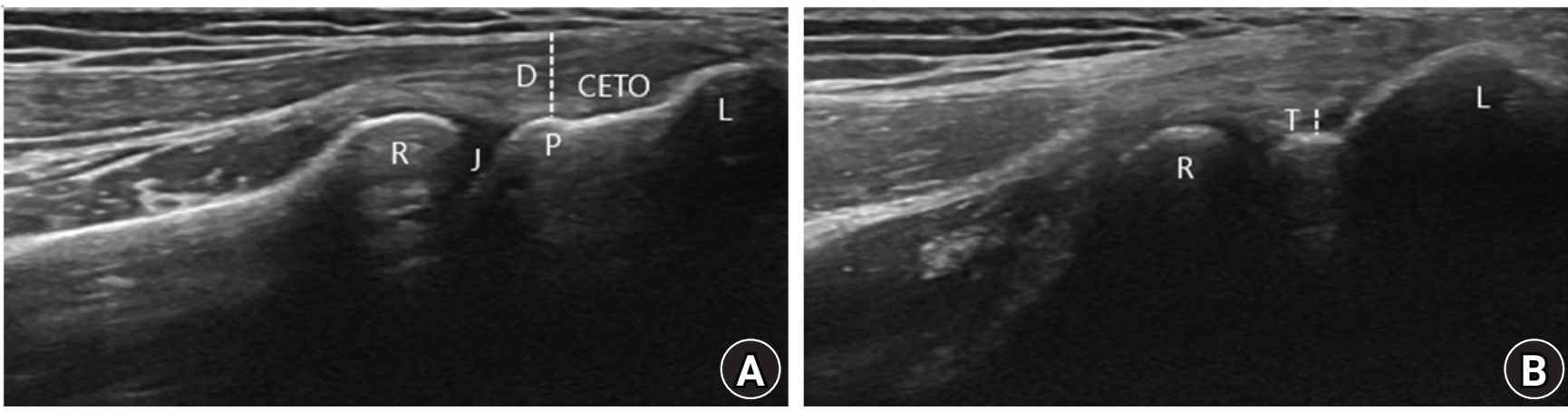

Fig. 3. The common extensor tendon depth (CETD) and the common extensor tendon tear thickness (CETTT) on longitudinal ultrasonographic examinations. (A) CETD. (B) CETTT. R: radial head, J: radiohumeral joint, D: dotted line indicates tendon depth, P: plateau, L: lateral epicondyle, CETO: common extensor tendon origin, T: CETTT.
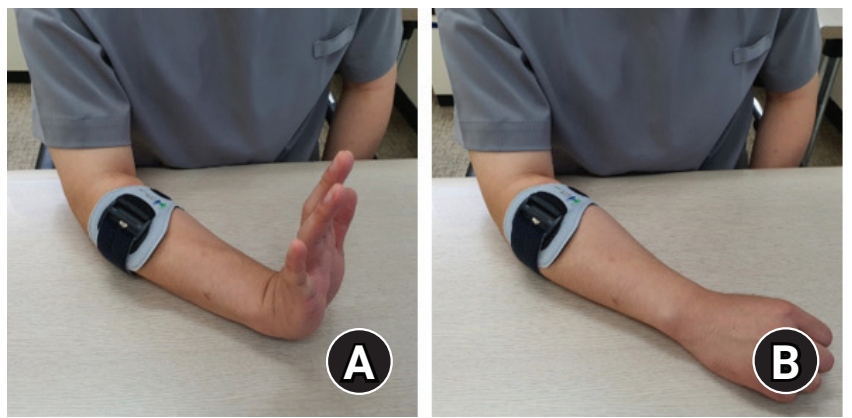

Fig. 4. Strengthening exercise of the extensor muscle of the elbow. (A) All the fingers and wrist were fully extended, and isometric force was applied for 10 seconds. (B) A period of rest was followed for 10 seconds with all the fingers slightly flexed and the wrist neutral. post hoc testing or Kruskal-Wallis analysis, followed by MannWhitney post hoc testing were used to analyze the values between the groups according to normality. The Mann-Whitney $\mathrm{U}$-test or independent t-test was performed to compare the values between the two groups according to normality. Statistical analysis was performed using IBM SPSS ver. 22 (IBM Corp., Armonk, NY, USA). A p $<0.05$ was considered statistically significant.

\section{RESULTS}

There are no significant differences in the demographic data of the three groups (Table 1). The overall functional scores and ul- 
Table 1. Demographic data (initial)

\begin{tabular}{lcccc}
\hline Variable & Group I $(\mathrm{n}=21)$ & Group II $(\mathrm{n}=21)$ & Group III $(\mathrm{n}=21)$ & $\mathrm{p}$-value \\
\hline Age $(\mathrm{yr})$ & $52.3 \pm 10.2(25-71)$ & $52.2 \pm 8.2(41-70)$ & $48.7 \pm 7.1(34-64)$ & 0.304 \\
Sex (male:female) & $11: 10$ & $13: 8$ & $9: 12$ & 0.472 \\
Dominant:nondominant & $14: 7$ & $16: 5$ & $14: 7$ & 0.743 \\
Symptom duration (wk) & $53.9 \pm 129.2(12-600)$ & $55.8 \pm 83.9(12-364)$ & $52.6 \pm 70.9(12-260)$ & 0.416 \\
No. of CS injections & $1.0 \pm 1.2(0-4)$ & $1.1 \pm 1.6(0-4)$ & $1.1 \pm 1.6(0-5)$ & 0.937 \\
VAS pain score (0-10) & $6.3 \pm 1.8(3.0-9.0)$ & $6.3 \pm 1.5(4.0-9.0)$ & $6.6 \pm 1.5(4.0-9.0)$ & 0.839 \\
MEPS (0-100) & $56.9 \pm 12.1(30.0-75.0)$ & $54.3 \pm 10.5(30.0-70.0)$ & $55.7 \pm 11.5(30.0-70.0)$ & 0.723 \\
CETD (cm) & $0.39 \pm 0.17(0.19-0.69)$ & $0.33 \pm 0.17(0.16-0.64)$ & $0.33 \pm 0.11(0.19-0.53)$ & 0.469 \\
CDA (grade, 0-4) & $1.9 \pm 0.7(1-4)$ & $1.6 \pm 0.8(1-4)$ & $1.7 \pm 0.8(1-4)$ & 0.336 \\
CETPTT (tear:no tear) & $11: 10$ & $11: 10$ & $9: 12$ & 0.779 \\
CETTT (cm) & $0.03 \pm 0.04(0.00-0.14)$ & $0.03 \pm 0.04(0.00-0.13)$ & $0.02 \pm 0.04(0.00-0.15)$ & 0.788 \\
\hline
\end{tabular}

Values are presented as mean \pm standard deviation (range). One-way analysis of variance or Kruskal-Wallis analysis was used to analyze the values among the three groups, $\mathrm{p}<0.05$.

CS: corticosteroid, VAS: visual analog scale, MEPS: Mayo elbow performance score, CETD: common extensor tendon depth measured at the "plateau" which is located at the origin of common extensor tendon and the humeroradial joint, CDA: color Doppler activity, CETPTT: common extensor tendon partial thickness tear, CETTT: common extensor tendon tear thickness.

Table 2. Overall functional and ultrasonographic outcome according to the three groups

\begin{tabular}{|c|c|c|c|}
\hline Variable & Initial & $12 \mathrm{wk}$ & p-value \\
\hline \multicolumn{4}{|l|}{ Group 1} \\
\hline VAS score $(0-10)$ & $6.3 \pm 1.8(3.0-9.0)$ & $3.6 \pm 1.9(0.0-7.0)$ & $<0.001$ \\
\hline MEPS (0-100) & $56.89 \pm 12.1(30.0-75.0)$ & $67.9 \pm 12.7(45.0-90.0)$ & 0.001 \\
\hline CETD $(\mathrm{cm})$ & $0.39 \pm 0.17(0.19-0.69)$ & $0.41 \pm 0.15(0.23-0.71)$ & 0.029 \\
\hline CDA (grade, 0-4) & $1.9 \pm 0.7(1-4)$ & $0.9 \pm 0.7(0-2)$ & 0.001 \\
\hline CETPTT (tear:no tear) & $11: 10$ & $11: 10$ & 1.000 \\
\hline $\operatorname{CETTT}(\mathrm{cm})$ & $0.03 \pm 0.04(0.00-0.14)$ & $0.03 \pm 0.04(0.00-0.14)$ & 0.005 \\
\hline \multicolumn{4}{|l|}{ Group 2} \\
\hline VAS score (0-10) & $6.3 \pm 1.5(4.0-9.0)$ & $2.3 \pm 2.0(0.0-6.0)$ & $<0.001$ \\
\hline MEPS (0-100) & $54.3 \pm 10.5(30.0-70.0)$ & $73.6 \pm 7.9(55.0-90.0)$ & $<0.001$ \\
\hline CETD $(\mathrm{cm})$ & $0.33 \pm 0.17(0.16-0.64)$ & $0.44 \pm 0.16(0.25-0.71)$ & $<0.001$ \\
\hline CDA (grade, 0-4) & $1.6 \pm 0.8(1-4)$ & $0.57 \pm 0.60(0-2)$ & 0.001 \\
\hline CETPTT (tear:no tear) & $11: 10$ & $9: 12$ & 0.157 \\
\hline CETTT $(\mathrm{cm})$ & $0.03 \pm 0.04(0.00-0.13)$ & $0.01 \pm 0.02(0.00-0.06)$ & 0.003 \\
\hline \multicolumn{4}{|l|}{ Group 3} \\
\hline VAS score $(0-10)$ & $6.6 \pm 1.5(4.0-9.0)$ & $3.2 \pm 2.0(1.0-8.0)$ & $<0.001$ \\
\hline MEPS (0-100) & $55.7 \pm 11.5(30.0-70.0)$ & $71.4 \pm 12.5(30.0-90.0)$ & $<0.001$ \\
\hline $\operatorname{CETD}(\mathrm{cm})$ & $0.33 \pm 0.11(0.19-0.53)$ & $0.41 \pm 0.10(0.25-0.55)$ & $<0.001$ \\
\hline CDA (grade, 0-4) & $1.7 \pm 0.8(1-4)$ & $0.7 \pm 0.8(0-3)$ & $<0.001$ \\
\hline CETPTT (tear:no tear) & $9: 12$ & $7: 14$ & 0.157 \\
\hline CETTT $(\mathrm{cm})$ & $0.02 \pm 0.04(0.00-0.15)$ & $0.02 \pm 0.03(0.00-0.09)$ & 0.012 \\
\hline
\end{tabular}

Values are presented as mean \pm standard deviation (range). The above analysis was performed using Wilcoxon signed-rank test or paired $t$-test according to the normality of data to evaluate the differences between initial and 12 weeks after treatment, $\mathrm{p}<0.05$.

VAS: visual analog scale score, MEPS: Mayo elbow performance score, CETD: common extensor tendon depth measured at the "plateau", CDA: color Doppler activity, CETPTT: common extensor tendon partial thickness tear, CETTT: common extensor tendon tear thickness.

trasonographic findings in all three groups except for the number of common extensor tendon partial tears improved after treatment (Table 2, Fig. 5). There are significant differences in the improvement of mean MEPS and CETD at 6 and 12 weeks among the three groups (MEPS: 6 weeks, $\mathrm{p}=0.039 ; 12$ weeks, $\mathrm{p}=0.022$; CETD: 6 weeks, $\mathrm{p}<0.001 ; 12$ weeks, $\mathrm{p}<0.001)$. The mean MEPS in group 2 significantly improved more than groups 1 and 3 at 6 weeks $(\mathrm{G} 1,56.9>62.4 ; \mathrm{G} 2,54.3>65.0 ; \mathrm{G} 3,55.7>$ 

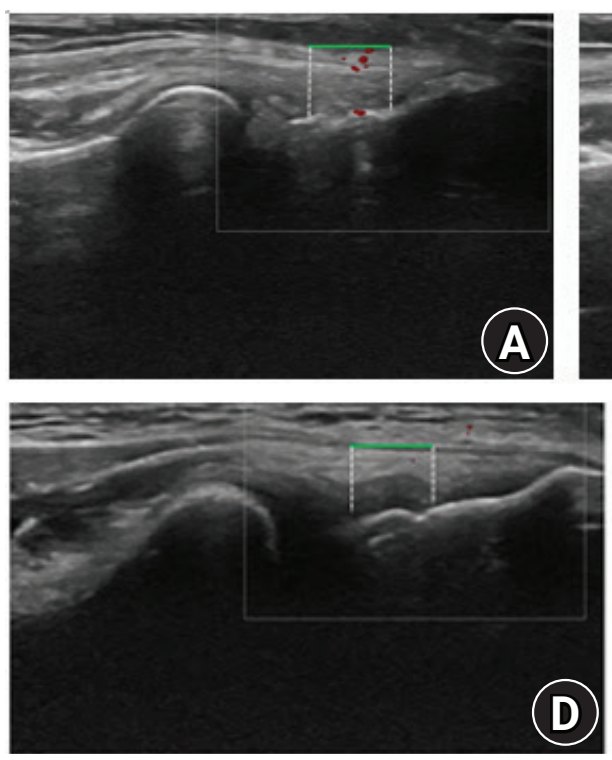
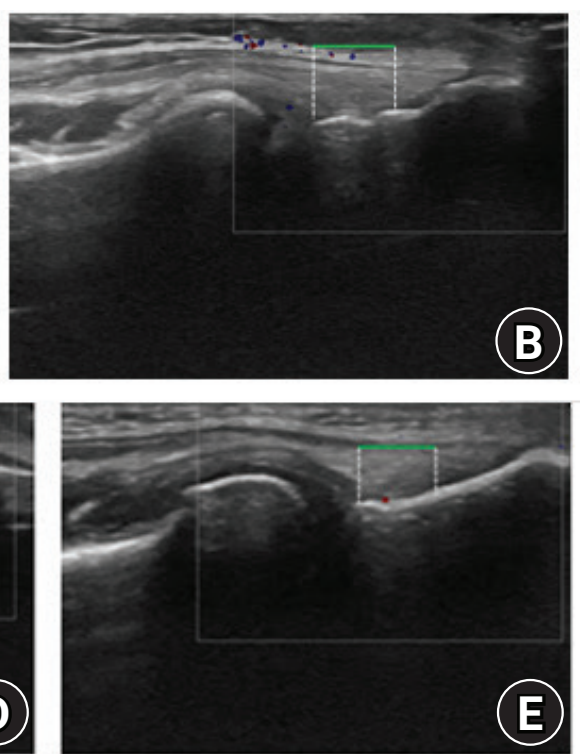
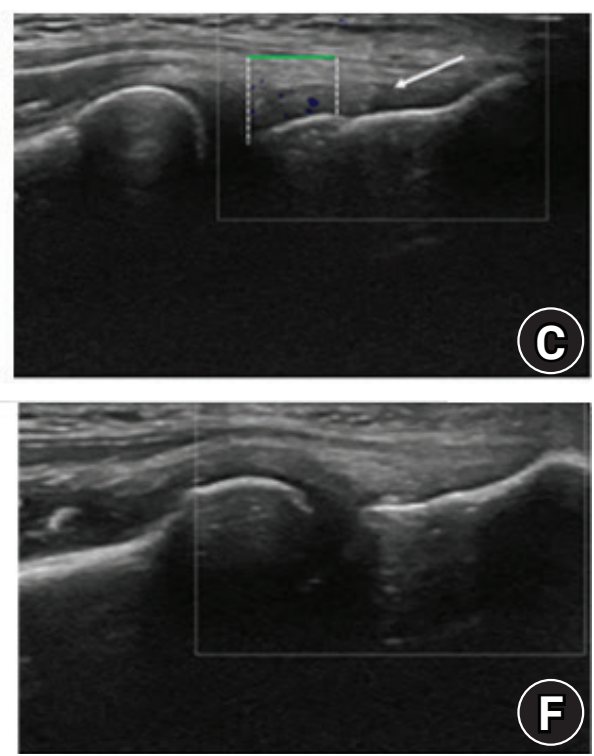

Fig. 5. The ultrasonographic follow-up of the three groups. (A) Group 1 at initial. Color Doppler activity (CDA), grade 2; common extensor tendon depth (CETD), 4.3. (B) Group 1 at 12 weeks. CDA, grade 2; CETD, $4.4 \mathrm{~mm}$. (C) Group 2 at initial. CDA, grade 2; CETD, $4.2 \mathrm{~mm}$. White arrow indicates a suspicion of a partial thickness tear of the common extensor tendon. (D) Group 2 at 12 weeks. CDA, grade 1; CETD, $4.5 \mathrm{~mm}$. (E) Group 3 at initial. CDA, grade 1; CETD, $3.8 \mathrm{~mm}$. (F) Group 3 at 12 weeks. CDA, grade 0; CETD, $4.0 \mathrm{~mm}$.

62.6; G1 vs. G2, $\mathrm{p}=0.036$; $\mathrm{G} 2$ vs. $\mathrm{G} 3, \mathrm{p}=0.039)$, and more than group 1 at 12 weeks $(\mathrm{G} 1,56.9>67.9 ; \mathrm{G} 2,54.3>73.6$; G1 vs. G2, $\mathrm{p}=0.018$ ). The mean CETD on ultrasonography in group 2 significantly increased more than groups 1 and 3 at 6 weeks (G1, $0.385>0.386$; G2, $0.332>0.392$; G3, $0.334>0.357$; G1 vs. G2, $\mathrm{p}<0.001$; G2 vs. G3, p < 0.001) and those in groups 2 and 3 increased more than group 1 at 12 weeks $(\mathrm{G} 1,0.385>0.409 ; \mathrm{G} 2$, $0.332>0.438$; $\mathrm{G} 3,0.334>0.405$ [cm]; $\mathrm{G} 1$ vs. $\mathrm{G} 2, \mathrm{p}<0.001 ; \mathrm{G} 1$ vs. $\mathrm{G} 3, \mathrm{p}=0.031)($ Table 3$)$.

\section{DISCUSSION}

Our results have shown that the mean VAS and MEPS of LE patients improved after EX only or combined with PDRN injections and ESWT. Ultrasonographic findings of LE patients including the mean CETD, CDA, and CETTT also improved after the treatments. Among the three groups, the mean MEPS in group 2 significantly improved more than groups 1 and 3 at 6 weeks, and more than group 1 at 12 weeks. The mean CETD on ultrasonography in group 2 significantly increased more than groups 1 and 3 at 6 weeks, and those in groups 2 and 3 increased more than group 1 at 12 weeks.

The treatment of LE is mainly conservative and encompasses physiotherapy, stretching or strengthening exercises, local injections (corticosteroid, PDRN, and PRP, etc.), and ESWT [1-3]. Although physical treatment modalities have been mentioned to be effective in the early period of treatment, their long-term effects are not definite [3]. Corticosteroid injections have been a frequently used treatment option, but its anti-inflammatory nature is reported to have only short-term to intermediate-term efficacies for pain relief. Therefore, the repetitive use of corticosteroids in LE is discouraged due to its adverse effects after the long-term use of steroid injections including tissue atrophy, tendon weakness, or tearing [10]. PRP is blood plasma with an increased concentration of autologous platelets, which is now being used as a part of wound treatment, bone healing, and muscle or tendon damage. It could potentially enhance tendon healing and tissue regeneration by delivering various growth factors and cytokines, thereby affecting cell proliferation, chemotaxis, cell differentiation, and angiogenesis $[2,11,12]$. There remains some debate about the efficacy of PRP for LE [12]. Systemic reviews that evaluated the effectiveness and safety of ESWT for the treatment of LE exist with conflicting results [13-16]. While the effects of ESWT in LE treatment have been found to be favorable in some studies $[13,14]$, other studies have mentioned certain local side effects like erythema, pain, and small hematomas [15,16]. PDRN is a tissue regeneration activator that is composed of a mixture of nucleotides and activates adenosine $\mathrm{A} 2 \mathrm{~A}$ receptors, stimulating VEGF and the activity of fibroblasts. PDRN has recently been used for the treatment of $\operatorname{LE}[5,6]$.

In the present study, CDA decreased in all three group after treatment because the inflammatory reactions gradually decreased. EX could strengthen the extensor muscle and tendon and stimulate its regeneration. Therefore, CETD significantly in- 
Table 3. Comparison of functional and ultrasonographic outcome among the three groups

\begin{tabular}{|c|c|c|c|c|c|c|}
\hline Group & VAS score & MEPS & CETD & CDA & CETPTT & CETTT \\
\hline \multicolumn{7}{|l|}{$6 \mathrm{wk}$} \\
\hline G1 vs. G2 & 0.835 & 0.036 & $<0.001$ & 0.893 & 0.152 & 0.054 \\
\hline G1 vs. G3 & 0.561 & 0.429 & 0.282 & 0.051 & 0.152 & 0.531 \\
\hline G2 vs. G3 & 0.768 & 0.039 & $<0.001$ & 0.048 & 1.000 & 0.721 \\
\hline One-way ANOVA or Kruskal-Wallis testing & 0.855 & 0.049 & $<0.001$ & 0.080 & 0.350 & 0.289 \\
\hline \multicolumn{7}{|l|}{$12 \mathrm{wk}$} \\
\hline G1 vs. G2 & 0.044 & 0.018 & $<0.001$ & 0.864 & 0.152 & 0.192 \\
\hline G1 vs. G3 & 0.251 & 0.328 & 0.031 & 0.638 & 0.152 & 1.000 \\
\hline G2 vs. G3 & 0.452 & 0.683 & 0.168 & 0.815 & 1.000 & 0.289 \\
\hline One-way ANOVA or Kruskal-Wallis testing & 0.138 & 0.022 & $<0.001$ & 0.906 & 0.350 & 0.383 \\
\hline
\end{tabular}

One-way analysis of variance (ANOVA), followed by Bonferroni post hoc testing or Kruskal-Wallis analysis, followed by Mann-Whitney post hoc testing was used to analyze the values between the groups according to normality, $\mathrm{p}<0.05$.

VAS: visual analog scale, MEPS: Mayo elbow performance score, CETD: common extensor tendon depth measured at the "plateau", CDA: color doppler activity, CETPTT: common extensor tendon partial thickness tear, CETTT: common extensor tendon tear thickness.

creased in all three groups after treatment. The effect of EX combined with PDRN injections or ESWT could increase compared to that of EX only. Compared to EX only or EX combined with ESWT, EX combined with PDRN exhibited a greater improvement in mean MEPS and CETD within the 12 weeks follow-up. The increase in the mean MEPS and CETD could be closely related to the aspect of tendon regeneration. The decrease in the mean CETTT in all three groups after treatment is also related with the tendon regeneration process. Although there is no significant difference among the three groups, two partial thickness tears of the common extensor tendon each in groups 2 and 3 were healed after the treatments.

EX is a conventional conservative treatment with a long history for LE. A combination therapy of several conservative treatments of LE could be more effective than a single therapy. There have been several attempts for combination therapy $[17,18]$. A single blinded randomized controlled trial reported that there were no significant differences amongst the physiotherapy, prolotherapy, and combined groups in the patient-related tennis elbow evaluation [17]. Another randomized controlled trial about braces versus physical therapy or a combination of both indicated that brace treatment may be useful as an initial therapy, and that combination therapy has no additional advantages compared to physical therapy, but is superior to braces only for the short term [18]. There are only a few studies about combination therapy, and further studies are necessary.

The present study has some limitations. First, there is no untreated control group, but this study is a randomized controlled trial about combination therapy. Combination therapy could be compared to single EX therapy. Second, the follow-up period is short-term. Since the follow-up is 12 weeks, this study cannot provide long-term results, but the functional and ultrasono- graphic parameters in this study could provide a definite comparison among the treated groups. Third, this study is single-blinded. Due to the nature of this study using injections and ESWT, this study was designed as a single-blinded randomized controlled trial. Fourth, the sample number is small, but the study satisfied a power analysis. Further considerations are necessary in the aspect of clinical significance. PDRN injections combined with EX exhibited a greater improvement in mean MEPS and mean CETD compared to EX only or EX combined with ESWT for LE within the 12 weeks follow-up.

\section{ACKNOWLEDGMENTS}

The authors wish to thank the orthopedic nurses, Yun-Il Choe, Byeong-Yi Choi, and Ye-Rin Yoon for checking the visual analog scale score and Mayo elbow performance score and helping with the ultrasonographic examinations.

\section{ORCID}

Bum Jin Shim

https://orcid.org/0000-0002-3751-2304

Eun-Min Seo

https://orcid.org/0000-0001-7964-9694

Jung-Taek Hwang

https://orcid.org/0000-0003-4189-084X

Do-Young Kim

https://orcid.org/0000-0003-3735-1640

Jae-Shin Yang

https://orcid.org/0000-0002-4142-709X

Su-Jung Seo

https://orcid.org/0000-0003-1667-9102

Myung Sun Hong

https://orcid.org/0000-0002-9264-2750

\section{REFERENCES}

1. Gündüz R, Malas FÜ, Borman P, Kocaoğlu S, Özçakar L. Physical therapy, corticosteroid injection, and extracorporeal shock 
wave treatment in lateral epicondylitis: clinical and ultrasonographical comparison. Clin Rheumatol 2012;31:807-12.

2. Krogh TP, Fredberg U, Stengaard-Pedersen K, Christensen R, Jensen P, Ellingsen T. Treatment of lateral epicondylitis with platelet-rich plasma, glucocorticoid, or saline: a randomized, double-blind, placebo-controlled trial. Am J Sports Med 2013; 41:625-35.

3. Kim GM, Yoo SJ, Choi S, Park YG. Current trends for treating lateral epicondylitis. Clin Shoulder Elb 2019;22:227-34.

4. Lee GJ, Park D. Usefulness of polydeoxyribonucleotide as an alternative to corticosteroids in patients with lateral epicondyitis: a case series. Medicine (Baltimore) 2018;97:e10809.

5. Altavilla D, Squadrito F, Polito F, et al. Activation of adenosine A2A receptors restores the altered cell-cycle machinery during impaired wound healing in genetically diabetic mice. Surgery 2011;149:253-61.

6. Galeano M, Bitto A, Altavilla D, et al. Polydeoxyribonucleotide stimulates angiogenesis and wound healing in the genetically diabetic mouse. Wound Repair Regen 2008;16:208-17.

7. Kwon DR, Park GY, Moon YS, Lee SC. Therapeutic effects of umbilical cord blood-derived mesenchymal stem cells combined with polydeoxyribonucleotides on full-thickness rotator cuff tendon tear in a rabbit model. Cell Transplant 2018;27:1613-22.

8. Dedes V, Tzirogiannis K, Polikandrioti M, Dede AM, Mitseas A, Panoutsopoulos GI. Comparison of radial extracorporeal shockwave therapy with ultrasound therapy in patients with lateral epicondylitis. J Med Ultrason (2001) 2020;47:319-25.

9. Nirschl RP, Kraushaar BS. Keeping tennis elbow at arm's length: simple, effective strengthening exercises. Phys Sportsmed 1996; 24:61-2.

10. Gulabi D, Uysal MA, Akça A, Colak I, Çeçen GS, Gumustas S.
USG-guided injection of corticosteroid for lateral epicondylitis does not improve clinical outcomes: a prospective randomised study. Arch Orthop Trauma Surg 2017;137:601-6.

11. Aspenberg P, Virchenko O. Platelet concentrate injection improves Achilles tendon repair in rats. Acta Orthop Scand 2004; 75:93-9.

12. Franchini M, Cruciani M, Mengoli C, et al. Efficacy of platelet-rich plasma as conservative treatment in orthopaedics: a systematic review and meta-analysis. Blood Transfus 2018; 16:502-13.

13. Stasinopoulos D, Johnson MI. Effectiveness of extracorporeal shock wave therapy for tennis elbow (lateral epicondylitis). Br J Sports Med 2005;39:132-6.

14. Haake M, König IR, Decker T, et al. Extracorporeal shock wave therapy in the treatment of lateral epicondylitis: a randomized multicenter trial. J Bone Joint Surg Am 2002;84:1982-91.

15. Chung B, Wiley JP. Effectiveness of extracorporeal shock wave therapy in the treatment of previously untreated lateral epicondylitis: a randomized controlled trial. Am J Sports Med 2004; 32:1660-7.

16. Ozkut AT, Kilinçoğlu V, Ozkan NK, Eren A, Ertaş M. Extracorporeal shock wave therapy in patients with lateral epicondylitis. Acta Orthop Traumatol Turc 2007;41:207-10.

17. Yelland M, Rabago D, Ryan M, et al. Prolotherapy injections and physiotherapy used singly and in combination for lateral epicondylalgia: a single-blinded randomised clinical trial. BMC Musculoskelet Disord 2019;20:509.

18. Struijs PA, Kerkhoffs GM, Assendelft WJ, Van Dijk CN. Conservative treatment of lateral epicondylitis: brace versus physical therapy or a combination of both-a randomized clinical trial. Am J Sports Med 2004;32:462-9. 